\title{
GSM AND GPS BASED MEDICAL EMERGENCY MODEL
}

\author{
Ramachandra Turkani ${ }^{1}$, Bharat Sukumar Biraj ${ }^{2}$ \\ ${ }^{1}$ Assistant Professor, KLE College of Engineering \& Technology, Chikodi, Karnataka, India \\ ${ }^{2}$ Student, ISE Department KLE College of Engineering \& Technology, Chikodi, Karnataka, India
}

\begin{abstract}
The medical emergency service is one of the most important needs of the human being in case any type of the diseases or the attacks. The recent numbers shows that there is a need in the service when we look at the rate of heart attacks. A first aid treatment could be given if it could be known as soon as possible after the attack. About 25\% of deaths occur in the age between 25-69 because of heart attacks And hence here we propose an idea to get the treatment or the emergency service for the heart attacks by sensing and sending the required information to the doctor and the listed people using GSM and with the location using GPS modems.
\end{abstract}

Keywords: GSM, GPS, Heart attack.

\section{INTRODUCTION}

There are among $48 \%$ of people in Mumbai are at risk of heart attack (27 sep. 2013 11:04 IST). About 25\% of deaths in the age of group 25-69 years occur because of heart attack. In urban areas, $32.8 \%$ deaths occur because of heart ailments, while this percentage in rural area is $22.9 \%$ [Times of India]. In order to make this cause to be under control we must learn how to detect the heart attack on time \& to get information about the person is going to have this kind of attack.

In order to detect the heart attack is not enough in the emergency cases. We have to inform the doctor about particular patient. It is very common thing that people will not take the precautions after doctor's prescriptions or warning also. In the much busy schedule of the common person, the patient is going to make his/her self busy in work. So it is the area that we have to inform the doctor and the relatives or the care takers of the patient that where the patient currently is and is there any emergency treatment needed.

In this paper we propose an efficient and effective system that to detect attack and the patient location, so this model is very useful for the people suffering with heart stroke. The proposed solution is based on mobile-cloud computing that does not require any modifications in signal infrastructure, while providing guidance in real time and being highly available. The paper explains about the introduction first and the related work which have carried out and proceeds with the proposed model and concludes with the conclusions and future work.

\section{HEART FAILURE}

Coronary artery disease (CAD) is the most common type and is the leading cause of heart attack. When you have CAD, your arteries become hard and narrow. Blood has a hard time getting to the heart, so the heart does not get all the blood it needs. CAD can lead to:

- Angina is discomfort that happens when the heart does not get enough blood. It may feel like a pressing or squeezing pain, often in the chest, but sometimes the pain is in the shoulders, arms, neck, jaw, or back. It can also feel like upset stomach. Angina is not a heart attack, but having angina means you are more likely to have a heart attack.

- Heart attack. A heart attack occurs when an artery is severely or completely blocked, and the heart does not get the blood it needs for more than 20 minutes.

A heart attack occurs when blood flow to a part of your heart is blocked for a long enough time that part of the heart muscle is damaged or dies. This means that other organs, which normally get blood from the heart, do not get enough blood. It does not mean that the heart stops. The medical term for this is myocardial infarction. A hard substance called plaque can build up in the walls of your coronary arteries. This plaque is made up of cholesterol and other cells. A troponin blood test can show if you have heart tissue damage. This test can confirm that you are having a heart attack. Signs of heart failure include:

- Feeling like you can't get enough air

- Swollen ankles and swollen feet

- Fatigue

- Stress-and anxiety

- Cough

- Fainting

- Dizziness

- Sweating

- Breathing- difficulty

- Your blood pressure may be normal, high, or low.

- Nausea 


\section{RELATED WORK}

The problem with detecting emergency status of the patient has been studied by many researchers working in the medical emergency technologies for the people or patients. Among some proposals, to solve this problem [1] is studied the currently used medical emergency and healthcare system in Malaysia. It also gives the drawback of sharing information between the hospitals. [2] Used the concept of system that provides an medical and healthcare information services to users via interactive search for personalized patient needs. [3]It produces an new method to optimize the distribution of firstaid resources. To rise the response speed in emergence medical services (EMS) extremely critical for the pre-hospital life saving. [4] Medical sensor networks allow for pervasive health monitoring in hospitals, at home, or on the way. Each user carries a set of wireless medical sensors that allows to monitor his health state.[5] Real-time information presents a persistent challenge to the emergency response community. In emergency situations, particularly with unconscious, incoherent and unaccompanied patients, providing emergency physicians with a patientpsilas accurate medical history could be the difference between life and death. [6] Delivering first aid before transferring the patient to the hospital is among keys to survival. Emergency message concept is used in our proposed system which is going to deliver the condition of the patient in emergency. Once we got the message from the patient, we can track his/her location very easily by using the GPS and GSM. [7] Describes an heart-attack self-test application for a mobile phones. Those based on mobile information processing.

The major problem with these proposals is that they are not able to detect the location of the patient and also they are not based on real time information processing. Their approach is on processing the information got from the patient in emergency cases. The application is going to work on some above dictated papers only when the user of that system is going to initiate that application. So we have to think of some different way to find the solution for the emergency situations for the persons. There was not any such application in which we that can be used at real time. A graph of heart attacks in India is rising and which needs a look in serious views. According to medical researchers there will be $25 \%$ of Indians going to suffer with heart attack in near of 2020.

So we thought that, this problem is going to be very large perspective in near future and we proposed this system. And the references say that these support an existing mobile information processing techniques.

\section{PROPOSED SYSTEM}

A system which is going to use an wearable sensors to detect heart attack [7].The sensors are mounted on the patient which will sense the parameters like blood pressure, sugar level, body temperature etc. The two main components are the mobile device with integrated GPS receiver and the compass, which could be any smart phone device in the market and the cloud server, which is basically the web service platform that can be employed to support a variety of context-awareness functionalities. The mobile device is responsible for local navigation, as well as interacting with the user to detect a heart attack based on some parameters.

There are some parameters used to detect the heart attack of a person. Those are heart rate, blood pressure, body temperature etc. The wearable sensors are used to detect the changes in the parameters in the body of the person. These sensors will transfer the information to the mobile device where this information is processed and going to generate a message in emergency cases. In emergency cases the system is going to process the information that has got form sensors then mobile device is going to process this information and generate a message to send to doctor and the relatives. Through the GPS receiver the location of the person is also going to get to the doctors only with that SMS.

It is also possibility that the person is not going to have any problem with the parameters detected by the sensor but he/she can have a chest pain in very high manner. So it is possible that this type is also going to cause a heart attack. To handle this type of conditions we introduced a user interface in the system. This interface may be one button which can be hold by the patient when they have the chest pain. Holding of this button for few seconds is going to generate an emergency message and processed above. The message can be conveyed to the doctors and the relatives of that person having severe heart attack.

SMS sent over the wireless area network from mobile station to destination mobile station which is multicast in nature. That is the SMS should be sent to doctor and relatives of the patient immediately. Multiple copies of SMS should be sent over the network, which is taken care by Emergency Response System shown in fig(1).

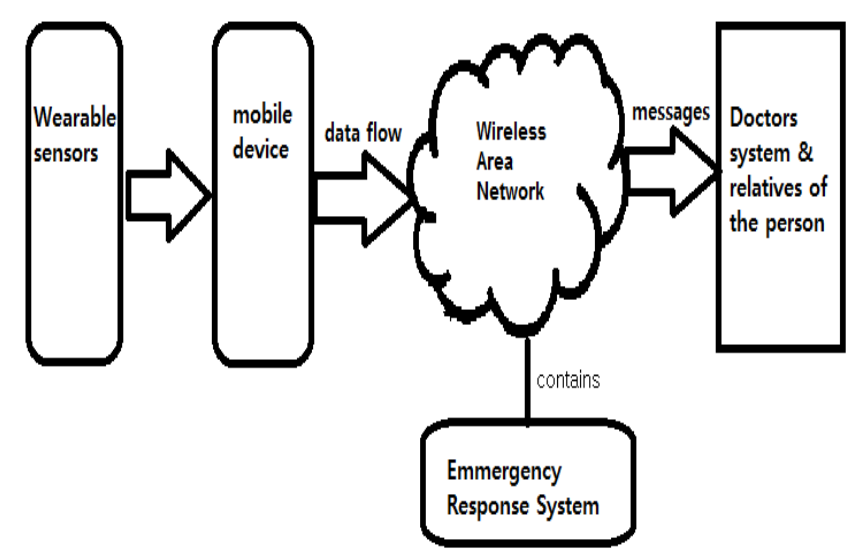

Fig 1: Emergency model. 
The values for parameters used to detect heart attack are collected in the mobile device which is near to the wearable sensors. This device is used to process gathered data from the sensors. The data fetched from the sensors should be accurate and fast real time data generation. After the processing of the data if the result is going to orient an severe situation then the message should be conveyed to doctors and the relative peoples of that person. So that they can provide the service to patient at emergency time and can minimize the severity of the heart attack.

Mobile device is going to send the position data. This data can be used to track the person or patients vary easily. The position data can be get in two ways: first by taking cell identification number of the mobile network and second by using GPS system to track global position of the device. The second approach is going to use a GPS satellites orbiting around the earth, which transmits signals that can be detected by anyone with GPS receiver. Using receiver, it is possible to detect the location of receiver.

\subsection{Working of System Components}

\subsubsection{Sensors}

The sensors should produce the data as fast as possible because the severity of the patient condition may rise at every second of time. So data generated by the sensors should be accurate and produce a real-time result of the attribute values defined for the parameters.

\subsubsection{Mobile Device}

This device is used to collect the information or parameters form the wearable sensors. These parameters values are processed and result is generated. If the result of parameters leads to the emergence of the patient condition then this information is transferred over the cloud. There is also an alternative situation where a person can have a high chest pain that time a person can interact with interface given with the system such as button. By interacting with system interface for example, by holding button more than 5 seconds, is going to generate emergency signal.

The mobile device can be used to track the person at the specific location by using the GPS and GSM. The person can be at any location the doctors and relatives are going to get the location information immediately with massages generated from the mobile device. So doctors and relatives can reach the patient very easily at there present location.

\subsubsection{Wireless Area Network}

The wireless area network is mainly used to have the conversation between the patient's and the doctor's devices. The message generated by mobile device of the patient has to deliver to the doctors. The main job of wireless network systems are to pass the information from one source system point of view to another destination system as early as possible and without any disturbance.

The network systems are going to have high rate of messages because a patient may changing his place at time. The wireless area network has to give highest priority to the emergency messages generated by the patient device. The system has to provide the location information, so we can track the patient vary easily. Therefore cloud has to pass the data form source to destination with very high speed.
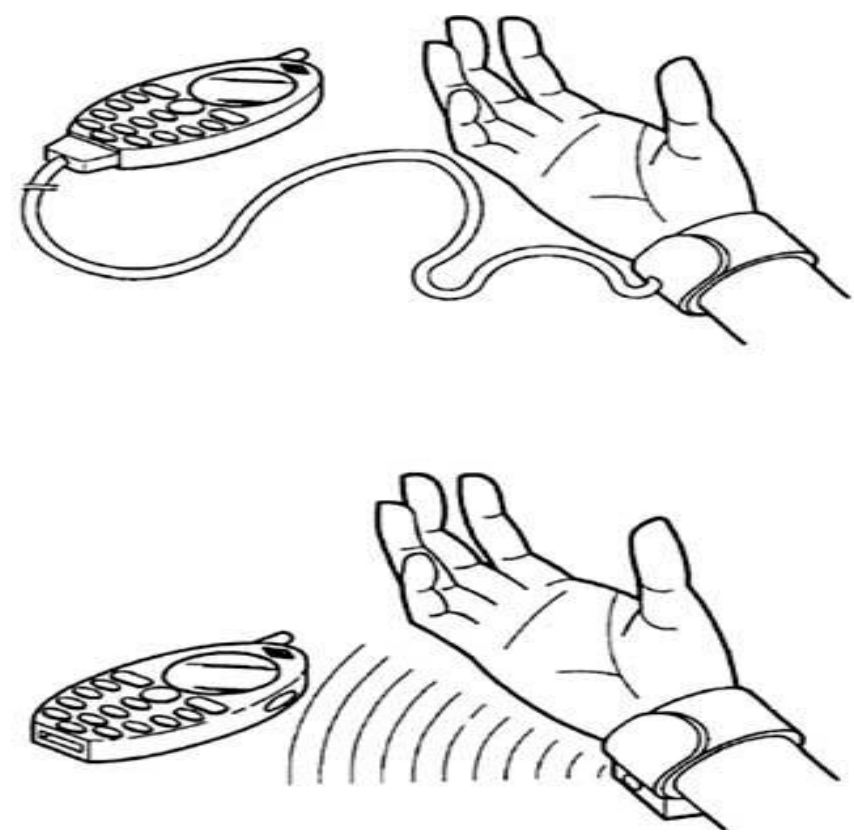

Fig 2: signal transmission of the measurer in different ways [9].

A mobile phone combined with physiological system comprises a physiological function signal receivable mobile phone, a measurer used to touch on the human skin and transmit detection signals to the mobile phone by wire or wireless transmission method, as shown in Fig. (2). The mobile phone can show measured data in its display for reminding a user of his health conditions [8].

The overall implementation is structured into three functional layers: sensing, communication and management. fig(3). Sensing layer implements a mobile monitoring for health data, signal processing, and data analysis. Communication layer includes short range wireless connection via Bluetooth, or different technologies for short range communication, and worldwide wireless connection via a mobile phone. Management layer carries out data processing and management tasks, mostly through internet. Researchers have proposed several systems based on the above three layers [8]. 


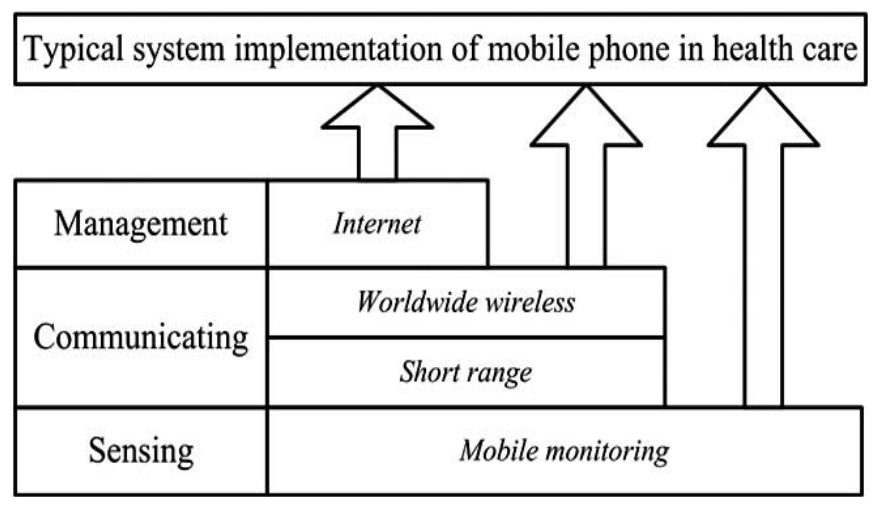

Fig 3: system implementation of mobile phone in health care.

Generally, the above inventions mainly comprise four modules: a user management module, managing user information: a data analysis module, analysing user's health condition based on the user information and health data stored in the memory and/or received from sensors and generating analysed result; a data management module, managing the user health data and the result, and a communicating module transmitting both.

\section{CONCLUSIONS AND FUTURE WORK}

The proposed system is giving an idea for medical emergency by using GPS and GSM. The system is going to use the data processing at client side so it provides a very high speed results and real-time operating on the produced information. The system is going to deliver the location information of the person so emergency service can reach patient as early as possible. The work of calculation or knowing the place of accident in high accuracy is to be done and correct parameters to be detected by the patient.

\section{REFERENCES}

[1] "Medical emergency and healthcare model: Enhancement with SMS and MMS facilities". Hammed S A, Miho V, Alkhateeb W, Hassan A Computer and Communication Engineering (ICCCE) ,2010.

[2] "A fast interactive search system for Healthcare services.” Daltayanni M, Chung Wang, Akella R. SRII Global Conference (SRII), 2012.

[3] "A new model for planning emergency facilities in Shaghai.” Qinyi Luo, Qiang Su, Jiajun Le, Linbin Lu. Service System and service Management (ICSSM), 2013.

[4] "Efficient and context-aware access control for pervasive medical sensor network". Garcia-Morchon O, Wehrle K. Pervasive Computing and Communication Workshop (PERCOM), 2010.

[5] "An RFID-Based System for emergency healthcare services". Turu C, Popa V. Advanced Information Networking and Applications Workshops, 2009.
[6] "Emergency SMS". Shirall-Shahreza M. SICE-ICASE, 2006.

[7] "A self-test to detect a heart attack using a mobile phone and wearable sensors". Leijdekkers P, Gay V. Computer-based Medical systems, 2008.

[8] "Mobile Phone Based Health Care Technology". Hao Wang and Jing Liu Bentham Science Publisher Ltd 2009 1874-7647/09 WAINA'09 